\title{
Biomarcadores en cirugía de aneurisma intracraneales no rotos: estudio piloto
}

\author{
Sebastián Paredes ${ }^{1}$, Javiera Benavides ${ }^{1,2}$, Alejandro Delfino ${ }^{1,2}$, Rommy von Bernhardi1,3
}

Introducción: La cirugía de exclusión de aneurisma no roto es un procedimiento de alto riesgo quirúrgico, con mortalidad de $2,6 \%$ y secuelas neurológicas permanentes de 10,9\%[1]. La interrupción transitoria del flujo sanguíneo cerebral (FSC) que se realiza durante la cirugía para disminuir el sangrado está asociado a complicaciones isquémicas hasta en un $26 \%$ de los pacientes[2]. Identificar biomarcadores asociados a esta interrupción transitoria del FSC podría predecir la aparición de déficit neurológico postoperatorio.

Objetivo: Identificar los niveles de biomarcadores neurológicos S-100 $\beta$, TGF $\beta$-1, tau y HSP70 en sangre y líquido cefalorraquídeo (LCR) durante distintos tiempos quirúrgicos previo y posterior al uso de interrupción transitoria del flujo sanguíneo cerebral.

Materiales y Métodos: Estudio piloto observacional prospectivo con aprobación del Comité de Ética de la Facultad de Medicina previo a su inicio. Nueve pacientes fueron reclutados durante un año para cirugía de aneurisma intracraneal no roto abierta de exclusión. La instalación de un catéter intratecal era decisión del equipo tratante y no se instaló para este estudio. Se excluyeron pacientes de cirugía de urgencia, traumatismo encefalocraneano o accidente cerebrovascular previo, enfermedades neurológicas degenerativas, demencia, neoplasias. Se utilizó un protocolo estandarizado de anestesia usando remifentanil y sevoflurano para mantención. Durante la interrupción transitoria del FSC se usó infusión de propofol para lograr tasas de supresión de 50\%. Las muestras de sangre y LCR se tomaron después de la inducción anestésica (T1), antes del primer clip transitorio (T2), después de 30 minutos del último clip transitorio (T3), después del cierre de piel (T4), 24 horas postoperatorias (T5), 48 horas postoperatorias (T6). Se usó test de Friedman para comparar los niveles de biomarcadores considerando significativo $p<0,05$. Post-hoc se realizó test de comparaciones múltiples de Dunn con corrección de Bonferroni.

Resultados: De los 9 pacientes un 33\% fueron hombres, edad media 49 años [rango 31 - 75] se obtuvieron 47 muestras de plasma y 10 muestras de LCR. El tiempo medio de clip transitorio fue 7,5 minutos [rango 3,5 - 25]. No hubo diferencias significativas en los biomarcadores durante los distintos tiempos de muestra en plasma ni LCR.

Conclusiones: No se encontró elevación significativa de S-100 $\beta$, TGF $\beta$-1, tau y HSP70 en sangre y LCR durante exclusión abierta de aneurismas intracraneanos con interrupción transitoria del FSC, lo que podría indicar ausencia de daño isquémico en esta muestra de pacientes.

\section{Referencias}

1. Raaymakers TW, Rinkel GJ, Limburg M, Algra A. Mortality and morbidity of surgery for unruptured intracranial aneurysms: a meta-analysis. Stroke. 1998 Aug;29(8):1531-8.

2. Ferch $R$, Pasqualin A, Pinna G, Chioffi F, Bricolo A. Temporary arterial occlusion in the repair of ruptured intracranial aneurysms: an analysis of risk factors for stroke. Journal of Neurosurgery [Internet]. Journal of Neurosurgery Publishing Group (JNSPG); 2002 Oct;836-42.

Pontificia Universidad Católica de Chile. Chile.

División Anestesiología. Chile.

Departamento Neurología. Chile. 